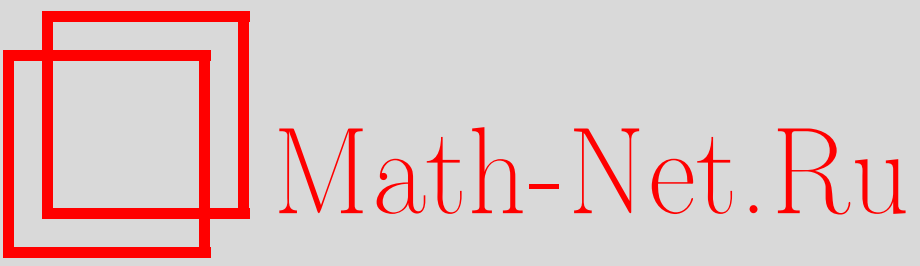

Ф. Калоджеро, Разрешимая задача трех тел и гипотезы Пенлеве, ТМФ, 2002, том 133, номер 2, 149-159

DOI: https://doi.org/10.4213/tmf386

Использование Общероссийского математического портала Math-Net.Ru подразумевает, что вы прочитали и согласны с пользовательским соглашением

http://www . mathnet.ru/rus/agreement

Параметры загрузки:

IP : 54.237 .59 .107

26 апреля 2023 г., 15:57:04 
ТЕОРЕТИЧЕСКАЯ

И МАТЕМАТИЧЕСКАЯ

ФИЗИКА

Том 133, № 2

ноябрь, 2002

(C) 2002 г.

Ф. Калоджеро*

\section{РАЗРЕШИМАЯ ЗАДАЧА ТРЕХ ТЕЛ И ГИПОТЕЗЫ ПЕНЛЕВЕ}

Показано, что при специальном выборе трех констант межчастичных взаимодействий в недавно привлекшем к себе внимание случае трех тел задачи многих тел в плоскости общее решение уравнений движения можно записать в замкнутом (и замечательно простом) виде. Обсуждается также другая аналогичная задача трех тел, и получены два сильно нелинейных автономных обыкновенных дифференциальных уравнения (ОДУ) третьего порядка, общее решение которых предположительно является целым, т.е. эти ОДУ должны обладать свойством Пенлеве (в сильном варианте).

Ключевые слова: задача трех тел, нелинейные обыкновенные дифференциальные уравнения, свойство Пенлеве.

\section{1. ВВЕДЕНИЕ}

В последнее время значительный интерес вызывала [1]-[3] следующая система, состояшая из $N$ обыкновенных дифференциальных уравнений (ОДУ):

$$
\zeta_{n}^{\prime \prime}=2 \sum_{\substack{m=1 \\ m \neq n}}^{N} a_{n m} \frac{\zeta_{n}^{\prime} \zeta_{m}^{\prime}}{\zeta_{n}-\zeta_{m}}, \quad n=1, \ldots, N .
$$

Зависимые переменные $\zeta_{n}=\zeta_{n}(\tau)$ являются комплексными, и интересно (см. ниже) установить их поведение, когда независимая переменная $\tau$ также комплексна. Штрихи обозначают производные по $\tau$. Константы взаимодействия $a_{n m}$ априори произвольны, за исключением требования симметрии

$$
a_{n m}=a_{m n}
$$

Три основные причины определяют интерес к этой системе ОДУ. Во-первых, полная интегрируемость этой системы в специальном случае, когда все константы взаимодействия равны единице, $a_{n m}=1$ [4], [2]. Во-вторых, при отождествлении комплексной $\zeta$-плоскости с физической плоскостью [5], [2] и при ограничении на вещественное

*Dipartimento di Fisica, Universitá di Roma "La Sapienza", Istituto Nazionale di Fisica Nucleare, Sezione di Roma, Roma, Italy.

E-mail: francesco.calogero@roma1.infn.it, francesco.calogero@uniroma1.it 
$\tau$ (интерпретируемое как “физическое время”) движение $N$ точек $\zeta_{n}$ соответствует решению задачи многих тел в плоскости, характеризуемой нюютоновскими уравнениями движения с несколькими интересными свойствами: парные силы, трансляционная и вращательная инвариантности, гамильтонова природа [1]-[3]. В-третьих, что, возможно, наиболее интересно в настоящем контексте, посредством замены независимой переменной [1]-[6]

$$
z_{n}(t)=\zeta_{n}(\tau), \quad \tau=\frac{e^{i \omega t}-1}{i \omega}
$$

систему (1) можно связать со следуюшей модифицированной системой ОДу:

$$
\ddot{z}_{n}=i \omega \dot{z}_{n}+2 \sum_{\substack{m=1 \\ m \neq n}}^{N} a_{n m} \frac{\dot{z}_{n} \dot{z}_{m}}{z_{n}-z_{m}}, \quad n=1, \ldots, N
$$

в которую входит дополнительная положительная константа $\omega$. Здесь точки обозначают производные по новой независимой переменной $t$, которую мы снова выбираем вещественной и интерпретируем как “физическое время” (в силу чего $\tau$ становится комплексной и перемешается против часовой стрелки по окружности $\widetilde{C}$, диаметр которой лежит в верхней половине комплексной $\tau$-плоскости, его нижний конец находится в начале координат $\tau=0$, а верхний конец - при $\tau=2 i / \omega ;$ см. (3)). Эту систему можно также связать с задачей многих тел в плоскости, которая, помимо того что обладает всеми указанными выше свойствами, характеризуется дополнительным присутствием одночастичной силы, действующей на каждую частицу и равной трехмерному векторному произведению скорости движушейся частицы (рассматриваемой как 3-вектор в плоскости, в которой происходит движение) и постоянного 3-вектора, ортогонального к этой плоскости. Следовательно, эту одночастичную силу можно интерпретировать как силу Лоренца, производимую постоянным магнитным полем, ортогональным к плоскости, в которой происходит движение, если предположить, что частицы наделены электрическим зарядом (одинаковым для всех частиц). Эффект этой внешней силы состоит в изгибе траекторий частиц, так что их движение становится замкнутым. Далее оказывается, что среди решений этой задачи многих тел имеется много решений с полностью периодическими траекториями. В самом деле, каждому решению системы (1), являющемуся мероморфным в замкнутом диске $C$, ограниченном окружностью $\widetilde{C}$, ясным образом соответствует (см. (3)) движение в задаче многих тел (4), являющееся полностью периодическим с периодом $T=2 \pi / \omega[1]-[3]$.

Этим определяется интерес к установлению аналитических свойств решений системы (1) и, в частности, к выделению тех специальных случаев (характеризуемых специальными значениями числа частиц $N$ и констант взаимодействия $a_{n m}$ ), при которых все решения $\zeta_{n}(\tau)$ системы (1) являются мероморфнымми функциями на (конечной части) комплексной $\tau$-плоскости, а именно к отождествлению систем (1), обладающих свойством Пенлеве (в формулировке для автономных систем). С физической точки зрения интерес к таким системам определяется соответствующим свойством задачи многих тел, характеризуемой уравнениями движения (4): все ее несингулярные траектории полностью периодические с периодом $T=2 \pi / \omega$ (в действительности, как указано 
выше, для этого достаточно, чтобы все решения соответствуюшей системы (1) были мероморфны внутри окружности $\widetilde{C}$, см. (3)). Но имеется также интерес к выделению таких случаев и с математической точки зрения, особенно когда это приводит (см. ниже) к отысканию одиночных ОДУ, которые (предположительно) обладают свойством Пенлеве.

В настоящей работе данная проблематика впервые рассматривается в контексте задачи трех тел $(N=3)$.

Как обсуждалось в работе [3], можно ожидать, что необходимое (если не достаточное) условие того, чтобы все решения $\zeta_{n}(\tau)$ системы (1) были мероморфными функциями $\tau$, состоит (в задаче трех тел, которой здесь ограничено наше рассмотрение) в иелочисленности (или бесконечности) всех семи показателей $\gamma_{n m}, \beta_{n m}, \Gamma$, определяемых в терминах трех констант взаимодействия $a_{n m}$ как

$$
\gamma_{n m}=\left(1+a_{n m}\right)^{-1}, \quad \beta_{n m}=-2 a_{n m}, \quad \Gamma=\frac{3}{3+2\left(a_{12}+a_{23}+a_{31}\right)}
$$

где индексы $n, m$ принимают значения $1,2,3$. Более того, этот подход подсказывает, что все решения $\zeta_{n}(\tau)$ системы (1) могут быть не только мероморфными, но и целыми, если все семь показателей (5), определяющихся значениями трех констант взаимодействия $a_{n m}$, являются неотрицательными целыми или бесконечными. (Показатели $\beta_{n m}$ существенны, только если их вещественные части положительны, $\operatorname{Re} \beta_{n m}>0$ [3], однако это ограничение несущественно в данном контексте, поскольку ниже мы ограничиваемся отрицательными значениями констант взаимодействия $\left.a_{n m}.\right)$

Интересно поэтому рассмотреть случаи, когда все семь определенных вьше чисел (см. (5) и (2)) являются целыми (включая нуль или бесконечность), а именно случаи, когда можно предположить, что все решения ОДУ (1) (при $N=3$ ) обладают свойством Пенлеве, а физические модели (4) обладают только полностью периодическими траекториями (за возможным исключением некоторых сингулярных траекторий, соответствуюших множеству начальных условий низшей размерности, которые, однако, могут появиться, только если некоторые из показателей (5) отрицательны).

Нетрудно выделить все значения констант взаимодействия, принадлежащие к определенной выше категории (заметим, что для целочисленности показателей $\gamma_{n m}$ константы взаимодействия $a_{n m}$ должны лежать в интервале $-2 \leqslant a_{n m} \leqslant 0$, а для целочисленности показателей $\beta_{n m}$ константы взаимодействия $a_{n m}$ должны сами быть целочисленными или полуцелочисленными; см. (5)). За исключением случаев, когда две из трех констант взаимодействия обрашаются в нуль (тогда задача сводится к задаче двух тел, а ее решения сводятся к квадратурам [3]), имеется всего 11 случаев (разумеется, с точностью до перестановок), приведенных в таблице (которую мы считаем достаточно наглядной, а потому не требуюшей подписи). 


\begin{tabular}{|c|c|c|c|c|c|c|c|c|c|c|}
\hline$a_{12}$ & 0 & 0 & 0 & 0 & 0 & 0 & $-1 / 2$ & $-1 / 2$ & $-1 / 2$ & $-1 / 2$ \\
\hline$a_{23}$ & $-1 / 2$ & $-1 / 2$ & $-1 / 2$ & -1 & -1 & $-3 / 2$ & $-1 / 2$ & $-1 / 2$ & $-1 / 2$ & $-1 / 2$ \\
\hline$a_{31}$ & $-1 / 2$ & -1 & $-3 / 2$ & -1 & -2 & $-3 / 2$ & $-1 / 2$ & -1 & -2 & $-3 / 2$ \\
\hline$\beta_{12}$ & 0 & 0 & 0 & 0 & 0 & 0 & 1 & 1 & 1 & 1 \\
\hline$\beta_{23}$ & 1 & 1 & 1 & 2 & 2 & 3 & 1 & 1 & 1 & 2 \\
\hline$\beta_{31}$ & 1 & 2 & 3 & 2 & 4 & 3 & 1 & 2 & 4 & 3 \\
\hline$\gamma_{12}$ & 1 & 1 & 1 & 1 & 1 & 1 & 2 & 2 & 2 & 2 \\
\hline$\gamma_{23}$ & 2 & 2 & 2 & $\infty$ & $\infty$ & -2 & 2 & 2 & 2 & $\infty$ \\
\hline$\gamma_{31}$ & 2 & $\infty$ & -2 & $\infty$ & -1 & -2 & 2 & $\infty$ & -1 & -2 \\
\hline$\Gamma$ & 3 & $\infty$ & -3 & -3 & -1 & -1 & $\infty$ & -3 & -1 & -1 \\
\hline
\end{tabular}

Заметим, что имеются три случая, когда все семь показателей $\gamma_{n m}, \beta_{n m}, \Gamma$ положительны или бесконечны; в этих случаях следует ожидать, что решения $\zeta_{n}(\tau)$ будут целыми функциями (в других восьми случаях они могут быть мероморфными - хотя такой вывод следовал бы из результатов работы [3], только если четыре показателя $\gamma_{n m}$ и Г были бы неотрицательны, что не имеет места в нашем случае).

В разделе 2 мы формулируем в более удобном виде ОДУ (1) для случая трех тел $(N=3)$. В разделе 3 мы даем в замкнутом (и замечательно простом) виде обшее решение для случая, соответствуюшего первому столбцу таблицы, когда одна константа взаимодействия обрашается в нуль, а две другие равны $-1 / 2, a_{12}=0, a_{23}=a_{31}=-1 / 2$; тем самым для данного случая мы подтверждаем сформулированные вьше ожидания. В разделе 4 для следуюшего случая в таблище (второй столбец: $a_{12}=0, a_{23}=-1 / 2$, $a_{31}=-1$ ) уравнения движения из раздела 2 редуцированы к двум одиночным (сильно нелинейным) ОДУ третьего порядка (см. ниже (35) и (41)), для которых тем самым предполагается справедливость свойства Пенлеве. Действительно, мы предполагаем, что их общее решение является целой функцией независимой переменной (на это указывает тот факт, что в данном случае все семь показателей (5) положительны и целочисленны или бесконечны, см. таблицу). Более простое из этих двух ОДУ третьего порядка также можно редуцировать к ОДУ четвертого порядка более стандартного типа (см. ниже (36)). Другие случаи из таблицы (в частности, еше один случай, когда ожидается наличие только целых решений, см. седьмой столбец в таблице) находятся в процессе изучения.

Добавлено при корректуре: Для случая, когда все константы связи равняются $-1 / 2$, теперь также получено решение, которое подтверждает это ожидание [7].

\section{2. ЗАДАЧА ТРЕХ ТЕЛ}

В данном разделе мы переформулируем в более удобном виде уравнения движения (1) при $N=3$. Прежде всего заметим, что, как ясно видно из (1), центр масс $\mathbf{Z} \equiv \mathbf{Z}(\tau)$,

$$
\mathbf{Z}=\frac{\zeta_{1}+\zeta_{2}+\zeta_{3}}{3}
$$

движется равномерно:

$$
\mathbf{Z}^{\prime \prime}=0, \quad \mathbf{Z}(\tau)=\mathbf{Z}(0)+\mathbf{Z}^{\prime}(0) \tau=\mathbf{Z}(0)+V \tau .
$$


Далее отметим существование (также очевидным образом следующее из (1) [3]) интеграла движения

$$
\widetilde{K}=\zeta_{1}^{\prime} \zeta_{2}^{\prime} \zeta_{3}^{\prime}\left(\zeta_{1}-\zeta_{2}\right)^{2 a}\left(\zeta_{2}-\zeta_{3}\right)^{2 b}\left(\zeta_{3}-\zeta_{1}\right)^{2 c} .
$$

Здесь и далее для удобства обозначений мы полагаем

$$
a_{12}=a_{21}=a, \quad a_{23}=a_{32}=b, \quad a_{31}=a_{13}=c .
$$

Введем теперь координаты $u_{n}$ относительно центра масс:

$$
u_{n}=\zeta_{n}-\mathbf{Z}, \quad n=1,2,3,
$$

которые, очевидно, удовлетворяют условию

$$
u_{1}+u_{2}+u_{3}=0,
$$

и (для удобства обозначений и в соответствии с (11)) положим

$$
u_{1}=x, \quad u_{2}=y, \quad u_{3}=-x-y .
$$

С помощью тривиальных алгебраических преобразований можно теперь записать уравнения движения (1) (при $N=3$ ) и интеграл движения $K=-(-1)^{-2 c} \widetilde{K}$ в терминах двух переменных $x$ и $y$ :

$$
\begin{aligned}
x^{\prime \prime} & =2 a \frac{\left(x^{\prime}+V\right)\left(y^{\prime}+V\right)}{x-y}-2 c \frac{\left(x^{\prime}+V\right)\left(x^{\prime}+y^{\prime}-V\right)}{2 x+y}, \\
y^{\prime \prime} & =-2 a \frac{\left(x^{\prime}+V\right)\left(y^{\prime}+V\right)}{x-y}-2 b \frac{\left(y^{\prime}+V\right)\left(x^{\prime}+y^{\prime}-V\right)}{2 y+x}, \\
K & =\left(x^{\prime}+V\right)\left(y^{\prime}+V\right)\left(x^{\prime}+y^{\prime}-V\right)(x-y)^{2 a}(x+2 y)^{2 b}(y+2 x)^{2 c},
\end{aligned}
$$

где $V=\mathbf{Z}^{\prime}(0)($ см. (7)).

\section{3. РАЗРЕШИМЫЙ СЛУЧАЙ}

В данном разделе мы приводим общее решение уравнений движения (13), (14) при $a=0, b=c=-1 / 2$ (что соответствует первому столбцу в приведенной выше таблице, см. (9)), а именно

$$
\begin{aligned}
& (y+2 x) x^{\prime \prime}=\left(x^{\prime}+V\right)\left(x^{\prime}+y^{\prime}-V\right), \\
& (x+2 y) y^{\prime \prime}=\left(y^{\prime}+V\right)\left(x^{\prime}+y^{\prime}-V\right) .
\end{aligned}
$$

Заметим, что обшее решение этой системы двух ОДУ второго порядка должно содержать четыре произвольные константы.

Предположим, что две функции $x(\tau), y(\tau)$ являются полиномами третьей степени по $\tau$; тогда они содержат восемь априори свободных параметров (каждый полином третьей степени имеет, разумеется, четыре коэффициента). Такой анзац очевидным образом совместим с (16), (17), и он преврашает эти два ОДУ в десять алгебраических уравнений 
(поскольку каждое из двух уравнений приравнивает два полинома четвертой степени, что влечет равенства их пяти коэффициентов). Получающаяся в результате система десяти алгебраических уравнений с восьмью неизвестными может показаться переопределенной; однако в действительности она не только может быть решена, но и оставляет четыре коэффициента неопределенными, тем самым гарантируя, что полученное таким образом решение действительно является обшим решением уравнений (16), (17). Это чудо кажется столь невероятным, что мы обнаружили его только непрямым способом, аналогичным тому, который мы используем в следующем разделе. Здесь же мы только приведем решение $(16),(17)$ в явном виде, оставляя усердному читателю (вполне несложную) задачу его проверки:

$$
\begin{gathered}
x(\tau)=L+M\left(\tau-\tau_{0}\right)+\frac{K}{6}\left(\tau-\tau_{0}\right)^{3} \\
y(\tau)=-\frac{L}{2}+(V-M)\left(\tau-\tau_{0}\right)+\frac{3}{4} \frac{L K}{V+M}\left(\tau-\tau_{0}\right)^{2}+\frac{K}{6}\left(\tau-\tau_{0}\right)^{3} .
\end{gathered}
$$

Здесь $L, M, K$ и $\tau_{0}$ - четыре произвольные константы; можно проверить, что $K$ совпадает с интегралом движения (15), который в данном случае имеет вид

$$
K=\frac{\left(x^{\prime}+V\right)\left(y^{\prime}+V\right)\left(x^{\prime}+y^{\prime}-V\right)}{(x+2 y)(y+2 x)} .
$$

Заметим, что этот результат (из которого, разумеется, следует, что все решения системы (1) являются в данном случае целыми, и соответственно все решения системы (4) в этом случае полностью периодические с периодом $T=2 \pi / \omega)$ согласован с ожиданиями, упомянутыми в конце раздела 1, поскольку в данном случае все показатели (5) или обращаются в нуль, или являются положительными целыми (см. первый столбец в таблице).

\section{4. ВЫВОД ДВУХ ОДУ, ОБШЕЕ РЕШЕНИЕ КОТОРЫХ ПРЕДПОЛАГАЕТСЯ ЦЕЛЫМ}

Нашей отправной точкой в данном разделе являются два связанньх ОДУ

$$
\begin{aligned}
& x_{2}=2 \frac{\left(x_{1}+V\right)\left(x_{1}+y_{1}-V\right)}{2 x+y}, \\
& y_{2}=\frac{\left(y_{1}+V\right)\left(x_{1}+y_{1}-V\right)}{2 y+x}
\end{aligned}
$$

и соответствуюшая константа интегрирования

$$
K=\frac{\left(x_{1}+V\right)\left(y_{1}+V\right)\left(x_{1}+y_{1}-V\right)}{(2 y+x)(2 x+y)^{2}},
$$

здесь и далее для удобства записи мы используем обозначения

$$
x_{j}=\frac{d^{j} x}{d \tau^{j}}, \quad y_{j}=\frac{d^{j} y}{d \tau^{j}}, \quad j=1,2,3,4 .
$$


Уравнения (21)-(24) отвечают уравнениям системы трех тел (13) и (14) при $a=0, b=$ $-1 / 2, c=-1$, а потому ожидается, что система будет обладать свойством Пенлеве, т.е. допускать только решения $x(\tau), y(\tau)$, являюшиеся целыми функциями комплексной переменной $\tau$, как обсуждалось в разделе 1 (см., в частности, второй столбец в таблице) и как подтверждается полной периодичностью с периодом $T=2 \pi / \omega$ всех решений соответствуюшей задачи трех тел (4), которые были получены численно [3].

Наша первая задача состоит в получении одного ОДУ для $y \equiv y(\tau)$. Логарифмическое дифференцирование уравнения (22) дает

$$
\begin{gathered}
\frac{y_{3}}{y_{2}}=\frac{y_{2}}{y_{1}+V}+\frac{x_{2}+y_{2}}{x_{1}+y_{1}-V}-\frac{2 y_{1}+x_{1}}{2 y+x}, \\
\frac{y_{3}}{y_{2}}=\frac{y_{2}}{y_{1}+V}+\frac{2\left(x_{1}+V\right)}{2 x+y}+\frac{y_{2}}{x_{1}+y_{1}-V}-\frac{2 y_{1}+x_{1}}{2 y+x}, \\
\frac{y_{3}}{y_{2}}=\frac{2\left(x_{1}+V\right)}{2 x+y}+\frac{y_{2}}{y_{1}+V}+\frac{y_{2}}{x_{1}+y_{1}-V}-\frac{y_{2}\left(2 y_{1}+x_{1}\right)}{\left(y_{1}+V\right)\left(x_{1}+y_{1}-V\right)}, \\
\frac{y_{3}}{y_{2}}=\frac{2\left(x_{1}+V\right)}{2 x+y} .
\end{gathered}
$$

Переход от (25) к (26) произведен с помошью (21) (чтобы исключить $x_{2}$ ), от (26) к (27) с помошью $(22)$ (для исключения знаменателя $(2 y+x)$ ), а переход к $(28)$ мы осушествляем, заметив, что три последних слагаемых в правой части (27) тождественно сокрашаются.

Возведем теперь $(28)$ в квадрат и исключим знаменатель $(2 x+y)^{2}$, используя $(23)$ :

$$
\left(\frac{y_{3}}{y_{2}}\right)^{2}=\frac{4 K\left(x_{1}+V\right)(2 y+x)}{\left(y_{1}+V\right)\left(x_{1}+y_{1}-V\right)} .
$$

Далее воспользуемся уравнением (22) для исключения члена $(2 y+x)$ :

$$
y_{3}^{2}=4 K y_{2}\left(x_{1}+V\right) \text {. }
$$

Более того, заметим, что из (23) с учетом (22) вытекает

$$
y_{2}\left(x_{1}+V\right)=K(2 x+y)^{2},
$$

а подстановка этой формулы в (30) дает

$$
y_{3}=2 K(2 x+y) \text {. }
$$

Но поскольку $2 x+y \equiv-3 y+2(x+2 y)$, то с помошью $(22)$ получаем

$$
2 x+y=-3 y+\frac{2\left(y_{1}+V\right)\left(x_{1}+y_{1}-V\right)}{y_{2}} .
$$

Подстановка этой формулы в (32) дает

$$
y_{3}=2 K\left\{-3 y+\frac{2\left(y_{1}+V\right)\left[\left(x_{1}+V\right)+y_{1}-2 V\right]}{y_{2}}\right\},
$$


где удобным образом выделен член $\left(x_{1}+V\right)$. Теперь исключим этот член, используя (30), в результате чего окончательно получим

$$
y_{3}^{2}\left(y_{1}+V\right)-y_{3} y_{2}^{2}=2 K y_{2}\left[3 y_{2} y-2\left(y_{1}+V\right)\left(y_{1}-2 V\right)\right] .
$$

Это (автономное нелинейное) ОДУ третьего порядка, общее решение которого $y \equiv$ $y(\tau)$ мы предполагаем целой функцией (комплексной) независимой переменной $\tau$ (вероятно, новой трансцендентной функцией). Заметим, что в ОДУ (35) имеются две произвольные константы $V$ и $K$ (которые, коль скоро они не нулевые, можно, конечно, заменить единицей с помошью масштабного преобразования зависимых и независимых переменных). Следовательно, его обшее решение $y \equiv y(\tau)$ будет содержать все пять произвольных констант (а именно $V, K$ и три константы интегрирования).

Дифференцируя ОДУ (35) по независимой переменной $\tau$ и исключая затем константу $K$ с помощью (35), получаем следуюшее ОДУ четвертого порядка, несколько более стандартного типа:

$$
y_{4}=R\left(y_{3}, y_{2}, y_{1}, y ; V\right),
$$

где рациональная функция $R\left(y_{3}, y_{2}, y_{1}, y ; V\right)$ имеет вид

$$
R\left(y_{3}, y_{2}, y_{1}, y ; V\right)=\frac{y_{3}}{y_{2}} \frac{y_{3}\left[3 y_{2} y-\left(y_{1}+V\right)\left(y_{1}-2 V\right)\right]-y_{2}^{2}\left(y_{1}-2 V\right)}{3 y_{2} y-2\left(y_{1}+V\right)\left(y_{1}-2 V\right)},
$$

a $V$ - снова произвольная константа (которую, если она не равна нулю, можно опять заменить единицей с помощью подходящего масштабного преобразования зависимых и независимой переменных). Как и раньше, мы предполагаем, что обшее решение ОДУ (36), (37) есть целая функция независимой переменной; оно, конечно, содержит четыре произвольные константы (помимо $V$ ) и действительно совпадает с обшим решением уравнения (35), где $K$ рассматривается теперь как (произвольная) константа интегрирования.

Очевидно, что ОДУ (36) допускает элементарное решение

$$
y(\tau)=A+B \tau+C \tau^{2},
$$

которое содержит три произвольные константы $A, B, C$. Формула (38) дает также (вполне тривиальное) решение уравнения (35) при $K=0$, а также удовлетворяет $(21),(22)$ вместе с

$$
x(\tau)=D-V \tau, \quad D=-2 A+\frac{(B+V)(B-2 V)}{2 C} .
$$

Другое решение уравнений (21), (22) (которое включает (38) как предельный случай) дается формулой

$$
y(\tau)=A-V \tau+B e^{C \tau},
$$

где $A, B, C$ - снова произвольные константы. Эта функция $y(\tau)$ также является решением уравнения (35) при $K=0$ (в действительности она дает его обшее решение, вместе с элементарным решением (38)), но не соответствует никакому из решений уравнений (21), (22) (одновременное выполнение формулы (40) и равенства $K=0$, очевидно, несовместно с вьполненным выше переходом от $(21),(22)$ к (35); заметим, например, что 
из (30) следует требование, чтобы $y_{3}$ равнялось нулю, что имеет место для элементарного решения (38), но не для решения (40) при $C \neq 0$ ).

Аналогичное рассмотрение (подробнее см. приложение) приводит к следуюшему ОДУ третьего порядка:

$$
\begin{aligned}
8 x_{3}^{2}\left(x_{1}+V\right)^{2}\left(x_{1}-2 V\right)+2 x_{3}\left[-x_{2}^{2}\left(7 x_{1}-8 V\right)+9 k x_{1}\left(x_{1}+V\right) x\right]\left(x_{1}+V\right)= & \\
= & -3 x_{2}^{4}\left(2 x_{1}-V\right)+3 k x_{2}^{2}\left(x_{1}+V\right)\left(5 x_{1}+2 V\right) x+ \\
& +2 k x_{2}\left(x_{1}+V\right)^{2}\left(x_{1}+4 V\right)^{2}-9 k^{2}\left(x_{1}+V\right)^{3} x^{2} .
\end{aligned}
$$

Здесь $k \equiv-4 K$ и $V$ - две произвольные константы (которые, коль скоро они не нулевые, снова можно заменить единищей с помошью подходяшего масштабного преобразования), и мы снова предполагаем, что обшее решение $x(\tau)$ ОДУ (41) есть целая функция независимой переменной $\tau$. Как и раньше, интересный вопрос состоит в том, является ли это общее решение новой трансцендентной функцией.

Для $k=0$ ОДУ (41) принимает гораздо более простой вид:

$$
8 x_{3}^{2}\left(x_{1}+V\right)^{2}\left(x_{1}-2 V\right)-2 x_{3} x_{2}^{2}\left(7 x_{1}-8 V\right)\left(x_{1}+V\right)+3 x_{2}^{4}\left(2 x_{1}-V\right)=0,
$$

и, как легко проверить, его общее решение имеет вид

$$
x(\tau)=A+\frac{V\left(\tau-\tau_{0}\right)}{2}+\frac{3}{2} \frac{V}{B} \operatorname{sh}\left[B\left(\tau-\tau_{0}\right)\right],
$$

где $A, B, \tau_{0}$ - три произвольные константы. Выражение (43), действительно являюшееся целой функцией, также дает решение уравнений $(21),(22)$ вместе с

$$
y(\tau)=-2 A-V\left(\tau-\tau_{0}\right) .
$$

Дифференцируя (41) по независимой переменной $\tau$, получаем ОДУ четвертого порядка

$$
x_{4}=\frac{P\left(x_{3}, x_{2}, x_{1}, x ; k, V\right)}{\left[8 x_{3}(x+V)\left(x_{1}-2 V\right)-x_{2}^{2}\left(7 x_{1}-8 V\right)+9 k x_{1}\left(x_{1}+V\right) x\right]\left(x_{1}+V\right)},
$$

где $P\left(x_{3}, x_{2}, x_{1}, x ; k, V\right)$ - полином второй степени по $x_{3}$, пятой степени по $x_{2}$, четвертой степени по $x_{1}$, второй степени по $x$, второй степени по $k$ и четвертой степени по $V$. Вывод явного вида этого полинома является тривиальной задачей, которую можно оставить усердному читателю или более сообразительному приверженцу алгебраических вычислений на компютере. Замена константы $k$ в правой части ОДУ (45) на корень уравнения (41) (рассматриваемого как уравнение второго порядка на неизвестную $k$ ) дает ОДУ четвертого порядка стандартного вида

$$
x_{4}=Q\left(x_{3}, x_{2}, x_{1}, x ; V\right),
$$

где функция $Q\left(x_{3}, x_{2}, x_{1}, x ; V\right)$ (в отличие от $R\left(y_{3}, y_{2}, y_{1}, y ; V\right)$, см. (37)) не является рациональной (она содержит квадратные корни). Относительно этого ОДУ четвертого порядка (выписывание явного вида которого снова является вполне тривиальной задачей, которую можно перепоручить усердному читателю) также предполагается, что оно 
обладает общим решением, которое является целой функцией независимой переменной (в действительности оно совпадает с обшим решением уравнения (41), когда $k$ рассматривается как произвольная константа интегрирования).

В заключение подчеркнем, что ОДУ, которым удовлетворяют $y(\tau)$ и $x(\tau)$ (см. соответственно (35) и (41)), как следует из их вывода, связаны нетривиальными дифференциальными преобразованиями (см. (21), (22) и (23)).

\section{ПРИЛОЖЕНИЕ}

Рассмотрим подробнее вывод нелинейного ОДУ третьего порядка (41). Первый шаг состоит в логарифмическом дифференцировании (22):

$$
\frac{x_{3}}{x_{2}}=x_{2}\left[\left(x_{1}+V\right)^{-1}+\left(x_{1}+y_{1}-V\right)^{-1}\right]+\frac{y_{2}}{x_{1}+y_{1}-V}-\frac{2 x_{1}+y_{1}}{2 x+y} .
$$

В правой части этого ОДУ мы скомбинируем два члена в квадратных скобках, заметив, что в силу (21) половина полученного таким образом вклада сокрашается с последним членом в правой части, и исключим $y_{2}$ с помошью соотношения

$$
y_{2}=K(2 x+y)^{2}\left(x_{1}+V\right)
$$

вытекающего из (23) и (22). Это дает

$$
\frac{x_{3}}{x_{2}}\left(x_{1}+V\right)=\left[\frac{x_{2}}{2}\left(2 x_{1}+y_{1}\right)+K(2 x+y)^{2}\right] \frac{1}{\lambda},
$$

где для удобства обозначений введена величина

$$
\lambda=x_{1}+y_{1}-V
$$

из определения которой непосредственно вытекает, что

$$
\begin{aligned}
& y_{1}+V=2 V-x_{1}+\lambda, \\
& 2 x_{1}+y_{1}=x_{1}+V+\lambda .
\end{aligned}
$$

Теперь заметим, что из (21) и (П.4а) следует

$$
2 x+y=\frac{2 \lambda\left(x_{1}+V\right)}{x_{2}} .
$$

С помощью этой формулы и (П.4в) мы получаем из (П.3), что

$$
\frac{x_{3}}{x_{2}}\left(x_{1}+V\right)=\frac{x_{2}}{2} \frac{x_{1}+V+\lambda}{\lambda}+4 K x_{2}^{-2}\left(x_{1}+V\right)^{2} \lambda .
$$

Это удобно переписать следующим образом:

$$
\frac{8 K \lambda^{2}\left(x_{1}+V\right)^{2}}{x_{2}}=\lambda\left[2 x_{3}\left(x_{1}+V\right)-x_{2}^{2}\right]-x_{2}^{2}\left(x_{1}+V\right) .
$$


С другой стороны, поскольку очевидно, что $x+2 y \equiv-3 x+2(2 x+y)$, из (П.5а) получается

$$
x+2 y=-3 x+\frac{4 \lambda\left(x_{1}+V\right)}{x_{2}},
$$

и подставляя выражения (П.4б), (П.4а), (П.5б) и (П.5а) в определение $K(23)$, мы получаем

$$
K=\frac{\left(x_{1}+V\right)\left(2 V-x_{1}+\lambda\right) \lambda}{\left[-3 x+4 \lambda\left(x_{1}+V\right) / x_{2}\right]\left[2 \lambda\left(x_{1}+V\right) / x_{2}\right]^{2}},
$$

что удобно переписать следующим образом:

$$
\frac{16 K \lambda^{2}\left(x_{1}+V\right)^{2}}{x_{2}}=\lambda\left[12 K\left(x_{1}+V\right) x+x_{2}^{2}\right]+x_{2}^{2}\left(2 V-x_{1}\right) .
$$

Сравнение (П.6б) и (П.7б) приводит к соотношению

$$
\lambda\left[4 x_{3}\left(x_{1}+V\right)-12 K\left(x_{1}+V\right) x-3 x_{2}^{2}\right]=x_{2}^{2}\left(4 V+x_{1}\right),
$$

которое дает

$$
\lambda=\frac{x_{2}^{2}\left(4 V+x_{1}\right)}{4 x_{3}\left(x_{1}+V\right)-12 K\left(x_{1}+V\right) x-3 x_{2}^{2}} .
$$

Наконец, подстановка этого выражения в (П.7б) дает нелинейное ОДУ

$$
\begin{array}{rl}
16 & K x_{2}\left(x_{1}+V\right)^{2}\left(4 V+x_{1}\right)^{2}=\left[4 x_{3}\left(x_{1}+V\right)-12 K\left(x_{1}+V\right) x-3 x_{2}^{2}\right] \times \\
& \times\left\{\left(4 V+x_{1}\right)\left[12 K\left(x_{1}+V\right) x+x_{2}^{2}\right]+\right. \\
\left.\quad+\left(2 V-x_{1}\right)\left[4 x_{3}\left(x_{1}+V\right)-12 K\left(x_{1}+V\right) x-3 x_{2}^{2}\right]\right\},
\end{array}
$$

которое с точностью до тривиальных преобразований совпадает с уравнением (41).

Благодарности. Мне приятно отметить полезные беседы с Робертом Конте, Овидиу Костином, Налини Йоши и Маттео Зоммакалом главным образом в ходе конференции "NEEDS 2001" и особенно с Мартином Крускалом как в ходе конференции, так и в течение моего последуюшего четырехнедельного пребывания в Институте математических наук Исаака Ньютона в сентябре-октябре 2001 г. в рамках семестра, посвяшенного интегрируемым системам.

\section{Список литературы}

[1] F. Calogero, J.-P. Françoise. Inverse Problems. 2001. V. 17. P. 1-8.

[2] F. Calogero. Classical Many-Body Problems Amenable to Exact Treatments. Lect. Notes in Phys. Monograph. V. 66. Berlin: Springer, 2001.

[3] F. Calogero, J.-P. Françoise, M. Sommacal. Periodic solutions of a many-rotator problem in the plane. II. Analysis of various motions. J. Nonlinear Math. Phys. (in press).

[4] F. Calogero. Nuovo Cimento B. 1978. V. 43. P. 177-241; Physica D. 2001. V. 152-153. P. $78-84$.

[5] F. Calogero. J. Math. Phys. 1998. V. 39. P. 5268-5291.

[6] F. Calogero. J. Math. Phys. 1997. V. 38. P. 5711-5719.

[7] F. Calogero. Solution of a three-body problem in the plane. Phys. Lett. A (submitted). 